\title{
Practical asymptotic stability of nonlinear stochastic evolution equations
}

\author{
Tomás Caraballo $^{a} \quad$ Mohamed Ali Hammami $^{b} \quad$ Lassaad Mchiri $^{b}$ \\ ${ }^{a}$ Universidad de Sevilla, \\ Dpto. Ecuaciones Diferenciales y Análisis Numérico, \\ Facultad de Matemáticas, Sevilla (Spain) \\ ${ }^{b}$ University of Sfax, Faculty of Sciences of Sfax, Department of Mathematics, Tunisia \\ \{E.mail: MohamedAli.Hammami@fss.rnu.tn\}
}

\begin{abstract}
In this paper we establish some sufficient conditions ensuring almost sure practical asymptotic stability with a non-exponential decay rate for solutions to stochastic evolution equations based on Lyapunov techniques.
\end{abstract}

Keywords: Stochastic evolution equations, Lyapunov techniques, almost sure practical asymptotic stability, decay function.

\section{Introduction}

The theory of stochastic differential equations in infinite dimensional spaces is already an established area of research, although the corresponding study of stability properties of solutions only experienced a rapid growth in the last two decades. In particular, most of the existing results are scattered throughout research journals and conference proceedings. These results have been obtained by using various methods, concepts and theorems from functional analysis, stochastic partial differential equations and functional differential equations. This makes it difficult for a newcomer to enter this interesting and important field. Several interesting and important variants to Lyapunov's original concepts of practical stability were proposed in [1]-[3]. When the origin is not necessarily an equilibrium point, we can study the asymptotic stability of solutions with respect to a small neighborhood of the origin. We would like to mention here the references [21], [22], among others. The asymptotic behaviour of systems described by stochastic evolution equations is a very important topic as the vast literature on this field shows. Moreover, in the nonlinear and/or nonautonomous situations it may happen that the stability can be even superexponential (see [8]). A point to be noted is that, as far as we know, the works appeared in the 
literature (see, for instance, [9]-[17] and [24]-[27]) are concerned with the exponential stability and the stabilization problem i.e. if the deterministic or stochastic system is not stable, how can we add a noisy term such that the stochastically perturbed model becomes pathwise exponentially stable? However this type of results fails to be applied, for instance, when the deterministic model is nonautonomous and has a super-exponential instability. In this paper, we will study the almost sure practical stability of and will illustrate the theory with an application example. The organisation of the paper is as follows. In section 2 we introduce the basic notations and assumptions. In Section 3 we prove some sufficient conditions ensuring almost sure practical stability of solutions to stochastic evolution equations, and study an example to illustrate these results.

\section{Basic notations and assumptions}

Let $V$ be a reflexible Banach space and $H$ a real separable Hilbert space such that

$$
V \subset H \equiv H^{*} \subset V^{*}
$$

where the injections are continuous and dense. In addition, we also assume both $V$ and $V^{*}$ are uniformly convex.

We denote by $\|\|,.|$.$| and \|.\|_{*}$ the norms in $V, H$ and $V^{*}$ respectively, by $(.,$.$) the inner$ product in $H$, and by $<., .>$ the duality between $V$ and $V^{*}$.

Assume $\{\Omega, \mathcal{F}, \mathbb{P}\}$ is a complete probability space with a normal filtration $\left\{\mathcal{F}_{t}\right\}_{t \geq 0}$, i.e. $\mathcal{F}_{0}$ contains the null sets in $\mathcal{F}$ and $\mathcal{F}_{t}=\cap_{s>t} \mathcal{F}_{s}$, for all $t \geq 0$, and let us consider a real valued $\left\{\mathcal{F}_{t}\right\}$-Wiener process $\{W(t)\}_{t \geq 0}$.

We denote by $I^{p}(0, T, V)$ (for $\left.p \geq 2\right)$ the closed subspace of $L^{p}(\Omega \times(0, T), \mathcal{F} \otimes \mathcal{B}([0, T]), \mathcal{P} \otimes$ $d t ; V)$ of all stochastic processes which are $\mathcal{F}_{t}$-adapted for almost every $t$ in $(0, T)$ (in what follows, a.e.t), where $\mathcal{B}([0, T])$ denotes the Borel $\sigma$-algebra of subsets in $[0, T]$. We write $L^{2}(\Omega ; C(0, T ; H))$ instead of $L^{2}(\Omega, \mathcal{F}, \mathcal{P} ; C(0, T ; H))$, where $C(0, T ; H)$ denotes the space of all continuous functions from $[0, T]$ into $H$.

In this article, we shall consider the following infinite-dimensional stochastic differential equation in $V^{*}$ and, for $T>0$,

$$
\left\{\begin{aligned}
d X(t) & =f(t, X(t)) d t+g(t, X(t)) d W_{t}, \quad t \in[0, T] \\
X(0) & =X_{0}
\end{aligned}\right.
$$

where $f(t,):. V \rightarrow V^{*}$ is a suitable family of (nonlinear) operators (see conditions below), $g(t,):. V \rightarrow H$ is another family of operators satisfying

(g1) The map $t \mapsto g(t, x)$ is Lebesgue measurable from $(0, T)$ into $H$, for all $x \in V$.

(g2) There exists $L>0$ such that

$$
|g(t, x)-g(t, y)| \leq L\|x-y\|, \quad \text { for all } x, y \in V, \quad \text { a.e.t. }
$$


and $X_{0} \in L^{p}\left(\Omega, \mathcal{F}_{0}, \mathcal{P} ; V\right)$ is an arbitrarily fixed initial datum.

As we are mainly interested in the stability analysis, we shall then assume that, for each $T>0$ and every $X_{0} \in L^{p}\left(\Omega, \mathcal{F}_{0}, \mathcal{P} ; V\right)$, there exists a process

$$
X(t) \in I^{p}(0, T, V) \cap L^{2}(\Omega ; C(0, T ; H)),
$$

which is solution to equation (3.5). In other words, $X(t)$ satisfies the following equation in $V^{*}$ :

$$
X(t)=X_{0}+\int_{0}^{t} f(s, X(s)) d s+\int_{0}^{t} g(s, X(s)) d W_{s}, \quad \forall t \in[0, T], \mathbb{P}-\text { a.s. }
$$

To this end, if we assume conditions below, we then can ensure that there exists a unique solution to the equation (2.1) (see [23]):

1. Measurability: for every $x \in V$, the map $t \in(0, T) \mapsto f(t, x) \in V^{*}$ is Lebesgue measurable, a.e. $t$.

2. Hemicontinuity: The map $\theta \in \mathbb{R} \mapsto<f(t, x+\theta y), z>\in \mathbb{R}$ is continuous for every $x, y, z \in V$, a.e. $t$.

3. Boundedness: There exists $\beta>0, c>0$ such that

$$
\|f(t, x)\|_{*} \leq c\|x\|^{p-1}+\beta \quad \text { for all } x \in V \text {, a.e.t. }
$$

4. Coercivity: There exist $\alpha>0, \lambda, \gamma \in \mathbb{R}_{+}^{*}$ such that

$$
2<f(t, x), x>+\|g(t, x)\|^{2} \leq-\alpha\|x\|^{p}+\lambda|x|^{2}+\gamma \quad \text { for all } x \in V, \quad \text { a.e.t. }
$$

5. Monotonicity:

$$
\|g(t, x)-g(t, y)\|^{2} \leq \lambda|x-y|^{2}-(2<f(t, x)-f(t, y), x-y>) \quad \text { for all } x, y \in V \text {, a.e.t. }
$$

We give now the definition of the almost surely convergence of solutions to a small ball centered at the origin.

Definition 2.1. The ball $B_{r}:=\left\{x \in \mathbb{R}^{d} /|x| \leq r\right\}, r>0$ is said to be almost surely globally practically uniformly exponentially stable if:

For any $x_{0}$ such that $0<\left|x\left(t, t_{0}, x_{0}\right)\right|-r$, for all $t \geq t_{0} \geq 0$, it holds that

$$
\lim _{t \rightarrow \infty} \sup \frac{1}{t} \ln \left(\left|x\left(t, t_{0}, x_{0}\right)\right|-r\right)<0 \text {, a.s. }
$$

System (2.1) is said to be almost surely globally practically uniformly exponentially stable if there exists $r>0$ such that $B_{r}$ is almost surely globally practically uniformly exponentially stable.

For the existence and uniqueness of solution we have the following two theorems ( see [4], [5], [6] and [10]).

Theorem 2.1. (Uniqueness Theorem). Assume that the hypotheses 1-5 and $(g 1)-(g 2)$ hold, then there exists at most one solution process of (2.2) in $I^{p}(0, T, V) \cap L^{2}(\Omega ; C(0, T ; H))$. 
Theorem 2.2. (Existence Theorem). Assume that the hypotheses 1-5 and $(g 1)-(g 2)$ hold, then there exists a unique solution of (2.2) in $I^{p}(0, T, V) \cap L^{2}(\Omega ; C(0, T ; H))$.

In what follows, we will assume, at least, the assumptions ensuring that the integrals in equation (2.2) make sense.

On the other hand, let us define some operators which will be used later on jointly with the Itô formula.

Unless otherwise is stated, we will assume that $U(t, x)$ is a $C^{1,2}$-positive appropriate Lyapunov functional such that for any $x \in V$ and $t \in \mathbb{R}_{+}, U_{x}^{\prime}(t, x) \in V$, and satisfies some additional assumptions which enable us to apply the Itô formula for the process $X(t)$, solution to equation (2.2) (see [23]). We can then define operators $L$ and $\mathcal{Q}$ as follows: for $x \in V, t \in \mathbb{R}_{+}$

$$
L U(t, x)=U_{t}^{\prime}(t, x)+<U_{x}^{\prime}(t, x), f(t, x)>+\frac{1}{2}\left(U_{x x}^{\prime}(t, x) g(t, x), g(t, x)\right),
$$

and

$$
\mathcal{Q} U(t, x)=\left(U_{x}^{\prime}(t, x), g(t, x)\right)^{2}
$$

\section{Practical stability of stochastic evolution equation}

The following lemma is known as the exponential martingale inequality and will play an important role in some of the results in this paper.

Lemma 3.1. Assume $X(t)$ is a solution to equation (2.1). Suppose $g(t, x)$ satisfies conditions $(g 1)$ and $(g 2), U(t, x)$ is an appropriate Lyapunov functional and $T, \alpha, \beta$ are any positive constants. Then

$$
\mathbb{P}\left\{\sup _{0 \leq t \leq T}\left[\int_{0}^{t}\left(U_{x}^{\prime}(s, X(s)), g(s, X(s))\right) d W(s)-\frac{\alpha}{2} \int_{0}^{t} \mathcal{Q} U(s, X(s)) d s\right]>\beta\right\} \leq \exp (-\alpha \beta) .
$$

Proof. See [19], Lemma 3.8.1.

We will introduce a precise definition of almost sure practical stability with general decay function $\lambda(t)$. We would like to mention that the concept of stability with ploynomial decay rate was initially introduced by Mao (see [20]), in the finite dimensional framework. Then this concept was generalized to the stability with general decay rate (see [18], [22]) although under stronger assumptions on the decay function than the ones we impose here.

Definition 3.1. Let $\lambda(t)$ be a positive function defined for sufficiently large $t>0$, say $t \geq T>0$, and satisfying that $\lambda(t) \uparrow+\infty$ as $t \rightarrow+\infty$. The solution $X(t)$ to equation (2.1) (defined in the future, i.e. for $t$ large enough) is said to decay to zero almost surely with decay function $\lambda(t)$ and order at least $\gamma>0$, if there exists a function $r(\cdot)$ such that $r(t) \rightarrow 0$ as $t \rightarrow+\infty$, and its generalized Lyapunov exponent is less than or equal to $-\gamma$ with probability one, i.e.

$$
\lim _{t \rightarrow+\infty} \sup \frac{\ln (|X(t)|-r(t))}{\ln (\lambda(t))} \leq-\gamma, \quad \mathbb{P}-\text { a.s. }
$$

If in addition 0 is solution to equation (2.1), the zero solution is said to be almost surely practically asymptotically stable with decay function $\lambda(t)$ and order at least $\gamma$, if there exist $r(t) \rightarrow 0$ as 
$t \rightarrow+\infty$ such that every solution to equation (2.1) decays to zero almost surely with decay function $\lambda(t)$ and order at least $\gamma$.

Remark 3.2. Clearly, replacing in the above definition $\lambda(t)$ by $O\left(e^{t}\right)$ leads to the usual practical exponential stability definition which has been analyzed in [16] for stochastic ordinary differential equations.

Theorem 3.3. Let $U(t, x)$ be an appropriate Lyapunov functional. Assume that $X(t)$ is a solution to equation (2.1) satisfying that $|X(t)| \neq 0$ for all $t \geq 0$ and $\mathbb{P}$-a.s. provided $|X(0)| \neq 0$ $\mathbb{P}$-a.s. Assume that there exist two continuous functions $\varphi_{1}(t) \in \mathbb{R}, \varphi_{2}(t) \geq 0$, and constants $q \in \mathbb{N}^{*}, m \geq 0, \nu \geq 0, \mu \geq 0, \theta \in \mathbb{R}$ and two small constants $\varrho>0$ and $\gamma \geq 0$ such that

$$
\begin{gathered}
(a) \quad \lambda(t)^{m}|x|^{q} \leq U(t, x), \quad \text { for all }(t, x) \in \mathbb{R}_{+} \times V, \\
\text { (b) } L U(t, x) \leq \varphi_{1}(t) U(t, x)+\varrho, \quad \text { for all }(t, x) \in \mathbb{R}_{+} \times V, \\
(c) \quad \mathcal{Q} U(t, x) \geq \varphi_{2}(t) U^{2}(t, x)+\gamma, \quad \text { for all }(t, x) \in \mathbb{R}_{+} \times V, \\
\text { (d) } \lim _{t \rightarrow+\infty} \sup \frac{\int_{0}^{t} \varphi_{1}(s) d s}{\ln \lambda(t)} \leq \theta, \quad \lim _{t \rightarrow+\infty} \inf \frac{\int_{0}^{t} \varphi_{2}(s) d s}{\ln \lambda(t)} \geq 2 \nu, \\
\lim _{t \rightarrow+\infty} \sup \frac{\ln t}{\ln \lambda(t)} \leq \frac{\mu}{2}, \\
\text { (e) } \lim _{t \rightarrow+\infty} \sup \frac{t}{\ln \lambda(t)}=c \geq 0, \quad \sum_{k=1}^{q}|x|^{q-k}\left[\frac{\varrho}{\lambda(t)^{m}}\right]^{\frac{k-1}{q}} \geq 1, \quad \text { for all } t \geq 0 .
\end{gathered}
$$

Then, for every $\alpha \in(0,1)$, it follows

$$
\lim _{t \rightarrow+\infty} \sup \frac{\ln \left(|X(t)|-\left(\frac{\varrho}{\lambda(t)^{m}}\right)^{\frac{1}{q}}\right)}{\ln \lambda(t)} \leq-\left[m-\left(\frac{1}{\alpha} \mu+\theta-\nu(1-\alpha)+c\right)\right], \quad \mathbb{P}-\text { a.s. }
$$

In particular, if $m>\frac{1}{\alpha} \mu+\theta-\nu(1-\alpha)+c$, the solution $X(t)$ decays to zero almost surely with decay function $\lambda(t)$ and order at least

$$
\gamma_{\alpha}=\left[m-\left(\frac{1}{\alpha} \mu+\theta-\nu(1-\alpha)+c\right)\right] .
$$

Proof. Fix $X_{0}$ such that $\left|X_{0}\right| \neq 0 \mathbb{P}$-a.s. Then, if we assume that $\lambda(t)^{m}|x|^{q}-\varrho>0$ for all $t \geq 0$, we immediately have $\lambda(t)^{m}|x|^{q}-\varrho \leq \lambda(t)^{m}|x|^{q} \leq U(t, x)$. Now, observe that

$$
\begin{aligned}
\lambda(t)^{m}|x|^{q}-\varrho & =\lambda(t)^{m}\left(|x|^{q}-\frac{\varrho}{\lambda(t)^{m}}\right) \\
& =\lambda(t)^{m}\left(|x|^{q}-\left(\left(\frac{\varrho}{\lambda(t)^{m}}\right)^{\frac{1}{q}}\right)^{q}\right) \\
& =\lambda(t)^{m}\left(|x|-\left(\frac{\varrho}{\lambda(t)^{m}}\right)^{\frac{1}{q}}\right)\left(|x|^{q-1}+|x|^{q-2}\left(\frac{\varrho}{\lambda(t)^{m}}\right)^{\frac{1}{q}}+\ldots+\left(\frac{\varrho}{\lambda(t)^{m}}\right)^{\frac{q-1}{q}}\right) \\
& =\lambda(t)^{m}\left(|x|-\left(\frac{\varrho}{\lambda(t)^{m}}\right)^{\frac{1}{q}}\right) \sum_{k=1}^{q}|x|^{q-k}\left[\frac{\varrho}{\lambda(t)^{m}}\right]^{\frac{k-1}{q}} \\
& \geq \lambda(t)^{m}\left(|x|-\left(\frac{\varrho}{\lambda(t)^{m}}\right)^{\frac{1}{q}}\right) .
\end{aligned}
$$


Therefore, $\lambda(t)^{m}\left(|x|-\left(\frac{\varrho}{\lambda(t)^{m}}\right)^{\frac{1}{q}}\right) \leq U(t, x)$ and $\ln \left[\lambda(t)^{m}\left(|x|-\left(\frac{\varrho}{\lambda(t)^{m}}\right)^{\frac{1}{q}}\right)\right] \leq \ln (U(t, x))$. Thus $\ln \left[\left(|x|-\left(\frac{\varrho}{\lambda(t)^{m}}\right)^{\frac{1}{q}}\right)\right]+m \ln (\lambda(t)) \leq \ln (U(t, x))$.

Applying the Itô formula and taking into account our assumptions we have

$$
\begin{aligned}
& d(\ln (U(t, X(t))))=\frac{L U(t, X(t))}{U(t, X(t))} d t+\frac{\left(U_{x}^{\prime}(t, X(t)), g(t, X(t))\right.}{U(t, X(t))} d W_{t} \\
& -\frac{1}{2} \frac{\mathcal{Q} U(t, X(t))}{U^{2}(t, X(t))} d t \\
& \int_{0}^{t} d(\ln (U(s, X(s)))) d s=\int_{0}^{t} \frac{L U(s, X(s))}{U(s, X(s))} d s+\int_{0}^{t} \frac{\left(U_{x}^{\prime}(s, X(s)), g(s, X(s))\right.}{U(s, X(s))} d W_{s} \\
& -\frac{1}{2} \int_{0}^{t} \frac{\mathcal{Q} U(s, X(s))}{U^{2}(s, X(s))} d s \\
& \ln (U(t, X(t)))=\ln (U(0, X(0)))+\int_{0}^{t} \frac{L U(s, X(s))}{U(s, X(s))} d s+M(t) \\
& -\frac{1}{2} \int_{0}^{t} \frac{\mathcal{Q} U(s, X(s))}{U^{2}(s, X(s))} d s, \\
& \ln (U(t, X(t))) \leq \ln (U(0, X(0)))+\int_{0}^{t} \frac{\varphi_{1}(s) U(s, X(s))+\varrho}{U(s, X(s))} d s+M(t) \\
& -\frac{1}{2} \int_{0}^{t} \frac{\mathcal{Q} U(s, X(s))}{U^{2}(s, X(s))} d s \\
& \leq \ln (U(0, X(0)))+\int_{0}^{t} \varphi_{1}(s) d s+\int_{0}^{t} \frac{\varrho}{U(s, X(s))} d s+M(t) \\
& -\frac{1}{2} \int_{0}^{t} \frac{\mathcal{Q} U(s, X(s))}{U^{2}(s, X(s))} d s \\
& \leq \ln (U(0, X(0)))+\int_{0}^{t} \varphi_{1}(s) d s+\int_{0}^{t} \frac{\varrho}{\lambda(s)^{m}|x(s)|^{q}} d s+M(t) \\
& -\frac{1}{2} \int_{0}^{t} \frac{\mathcal{Q} U(s, X(s))}{U^{2}(s, X(s))} d s \\
& \ln \left(U(t, X(t)) \leq \ln (U(0, X(0)))+\int_{0}^{t} \varphi_{1}(s) d s+t+M(t)-\frac{1}{2} \int_{0}^{t} \frac{\mathcal{Q} U(s, X(s))}{U^{2}(s, X(s))} d s\right.
\end{aligned}
$$

where $M(t)=\int_{0}^{t} \frac{\left(U_{x}^{\prime}(s, X(s)), g(s, X(s))\right.}{U(s, X(s))} d W_{s}$ is a continuous martingale with initial value $M(0)=0$. Due to the exponential martingale inequality (see Lemma 3.1),

$$
\mathbb{P}\left\{\omega: \quad \sup _{0 \leq t \leq w}\left[M(t)-\frac{u}{2} \int_{0}^{t} \frac{\mathcal{Q} U(s, X(s))}{U^{2}(s, X(s))} d s\right]>\nu\right\} \leq \exp (-u \nu),
$$

for any positive constants $u, \nu$ and $w$. In particular, taking $0<\alpha<1$ and setting $u=\alpha$, $\nu=\frac{2}{\alpha} \ln (k-1), w=k, k=2,3, \ldots$ we can apply the Borel-Cantelli lemma to obtain that, for almost all $\omega \in \Omega$, there exists an integer $k_{0}(\epsilon, \omega)>0$ such that

$$
M(t) \leq \frac{2}{\alpha} \ln (k-1)+\frac{\alpha}{2} \int_{0}^{t} \frac{\mathcal{Q} U(s, X(s))}{U^{2}(s, X(s))} d s,
$$


for $0 \leq t \leq k, k \geq k_{0}(\epsilon, \omega)$. Substituting this into equation (3.1) and using condition (c), we arrive at

$$
\begin{aligned}
\ln (U(t, X(t)) \leq & \ln (U(0, X(0)))+\int_{0}^{t} \varphi_{1}(s) d s+t+M(t)-\frac{1}{2} \int_{0}^{t} \frac{\mathcal{Q} U(s, X(s))}{U^{2}(s, X(s))} d s \\
\leq & \ln (U(0, X(0)))+\frac{2}{\alpha} \ln (k-1)+\int_{0}^{t} \varphi_{1}(s) d s+t-\frac{(1-\alpha)}{2} \int_{0}^{t} \varphi_{2}(s) d s \\
& -\frac{(1-\alpha)}{2} \int_{0}^{t} \frac{\gamma}{U^{2}(s, X(s))} d s \\
\leq & \ln (U(0, X(0)))+\frac{2}{\alpha} \ln (k-1)+\int_{0}^{t} \varphi_{1}(s) d s+t-\frac{(1-\alpha)}{2} \int_{0}^{t} \varphi_{2}(s) d s
\end{aligned}
$$

for $0 \leq t \leq k, k \geq k_{0}(\epsilon, \omega)$. Now, using condition (d), it follows

$$
\ln \left(U(t, X(t)) \leq \ln (U(0, X(0)))+\frac{\mu+\epsilon}{\alpha} \ln \lambda(t)+(\theta+\epsilon) \ln \lambda(t)-\frac{(1-\alpha)}{2}(2 \nu-\epsilon) \ln \lambda(t)+t,\right.
$$

for $k-1 \leq t \leq k, k \geq k_{0}(\epsilon, \omega)$, which implies that, using condition (e),

$$
\lim _{t \rightarrow+\infty} \sup \frac{\ln U(t, X(t))}{\ln \lambda(t)} \leq \frac{\mu+\epsilon}{\alpha}+(\theta+\epsilon)-\frac{(1-\alpha)}{2}(2 \nu-\epsilon)+c, \quad \mathbb{P}-\text { a.s. }
$$

Noting that, for $t \geq 0$ and $q \in \mathbb{N}^{*}$,

$$
\ln \left(|X(t)|-\left(\frac{\varrho}{\lambda(t)^{m}}\right)^{\frac{1}{q}}\right) \leq \ln (U(t, X(t)))-m \ln (\lambda(t))
$$

and taking into account that $\epsilon>0$ is arbitrary, we deduce

$$
\lim _{t \rightarrow+\infty} \sup \frac{\ln \left(|X(t)|-\left(\frac{\varrho}{\lambda(t)^{m}}\right)^{\frac{1}{q}}\right)}{\ln \lambda(t)} \leq-\left[m-\left(\frac{1}{\alpha} \mu+\theta-\nu(1-\alpha)+c\right)\right], \quad \mathbb{P}-\text { a.s. },
$$

as required.

Remark 3.4. We would like to point out that, to find the optimal value $\gamma^{*}=\sup _{0<\alpha<1} \gamma_{\alpha}$, we need to find out the minimum value $f^{*}$ for the function $f(\alpha)=\frac{1}{\alpha} \mu+\theta-\nu(1-\alpha)+c$ when the paramter $\alpha \in(0,1)$, and consequently it will hold that $\gamma^{*}=\left(m-f^{*}\right)$. Now, by straightforward computations, it is not difficult to check that

$$
\left\{\begin{array}{l}
f^{*}=2(\mu \nu)^{\frac{1}{2}}+\theta-\nu+c, \quad \text { if } \quad 0 \leq \mu<\nu \\
f^{*}=\mu+\theta+c, \quad \text { if } \quad \nu \leq \mu
\end{array}\right.
$$

which implies that

$$
\left\{\begin{array}{l}
\gamma^{*}=m-\left[2(\mu \nu)^{\frac{1}{2}}+\theta-\nu+c\right], \quad \text { if } \quad 0 \leq \mu<\nu \\
\gamma^{*}=m-[\mu+\theta+c], \quad \text { if } \quad \nu \leq \mu
\end{array}\right.
$$

Now, we will improve the statement of Theorem 3.3 when $\mathcal{Q}$ is also bounded above. 
Theorem 3.5. Let $U(t, x) \in C^{1,2}\left(\mathbb{R}_{+} \times H ; \mathbb{R}^{*}\right)$ be an appropriate Lyapunov functional. Assume that there exist three continuous functions $\varphi_{1}(t) \in \mathbb{R}, \varphi_{2}(t) \geq 0, \varphi_{3}(t) \geq 0$ and constants $q \in \mathbb{N}^{*}$, $m \geq 0, \nu \geq 0, \mu \geq 0, \theta \in \mathbb{R}$ and two small constants $\varrho>0$ and $\gamma \geq 0$ such that

$$
\begin{gathered}
(a) \quad \lambda(t)^{m}|x|^{q} \leq U(t, x), \quad \text { for all }(t, x) \in \mathbb{R}_{+} \times V, \\
\text { (b) } L U(t, x) \leq \varphi_{1}(t) U(t, x)+\varrho, \quad \text { for all }(t, x) \in \mathbb{R}_{+} \times V, \\
(c) \quad \varphi_{2}(t) U^{2}(t, x)+\gamma \leq \mathcal{Q} U(t, x) \leq \varphi_{3}(t) U^{2}(t, x), \quad \text { for all }(t, x) \in \mathbb{R}_{+} \times V, \\
(d) \quad \lim _{t \rightarrow+\infty} \sup \frac{\int_{0}^{t} \varphi_{1}(s) d s}{\ln \lambda(t)} \leq \theta, \quad \lim _{t \rightarrow+\infty} \inf \frac{\int_{0}^{t} \varphi_{2}(s) d s}{\ln \lambda(t)} \geq 2 \nu, \\
\lim _{t \rightarrow+\infty} \sup \frac{\int_{0}^{t} \varphi_{3}(s) d s}{\ln \lambda(t)} \leq \mu . \\
\text { (e) } \lim _{t \rightarrow+\infty} \sup \frac{t}{\ln \lambda(t)}=c, \quad \sum_{k=1}^{q}|x|^{q-k}\left[\frac{\varrho}{\lambda(t)^{m}}\right]^{\frac{k-1}{q}} \geq 1, \quad \text { for all } t \geq 0 .
\end{gathered}
$$

Then,

$$
\lim _{t \rightarrow+\infty} \sup \frac{\ln \left(|X(t)|-\left(\frac{\varrho}{\lambda(t)^{m}}\right)^{\frac{1}{q}}\right)}{\ln \lambda(t)} \leq-[m-(\theta-\nu+c)], \quad \mathbb{P}-\text { a.s. }
$$

In particular, if $m>\theta-\nu+c$, the solution $X(t)$ decays to zero almost surely with decay function $\lambda(t)$ and order at least

$$
\gamma=m-(\theta-\nu+c)
$$

Proof. Fix again $X_{0}$ such that $\left|X_{0}\right| \neq 0 \mathbb{P}$-a.s., and assume that $\lambda(t)^{m}|x|^{q}-\varrho>0$, for all $t \geq 0$. Then, the Itô formula implies again that inequality (3.1) holds true. Using conditions (b) and (c) we obtain

$$
\ln (U(t, X(t))) \leq \ln (U(0, X(0)))+M(t)+\int_{0}^{t} \varphi_{1}(s) d s+t-\frac{1}{2} \int_{0}^{t} \varphi_{2}(s) d s .
$$

Now condition (d) and inequality (3.4) imply

$$
\ln (U(t, X(t))) \leq \ln (U(0, X(0)))+M(t)+(\theta+\epsilon) \ln \lambda(t)+t-\frac{1}{2}(2 \nu-\epsilon) \ln \lambda(t),
$$

and condition (e) imply

$$
\lim _{t \rightarrow+\infty} \sup \frac{\ln U(t, X(t))}{\ln \lambda(t)} \leq \lim _{t \rightarrow+\infty} \sup \frac{M(t)}{\ln \lambda(t)}+\theta+\epsilon-\frac{1}{2}(2 \nu-\epsilon)+c, \quad \mathbb{P}-\text { a.s. }
$$

Let us denote by $\langle M(t)\rangle$ the quadratic variation process associated to $M(t)$. From our assumptions, we can deduce that $M(t)$ is a local martingale vanishing at $t=0$. Moreover, condition (c) implies

$$
\int_{0}^{t} \varphi_{2}(s) d s+\int_{0}^{t} \frac{\gamma}{U^{2}(s, X(s))} d s \leq<M(t)>=\int_{0}^{t} \frac{\mathcal{Q} U(s, X(s))}{U^{2}(s, X(s))} d s \leq \int_{0}^{t} \varphi_{3}(s) d s .
$$


Now, as $\nu>0$, it follows that $\lim _{t \rightarrow+\infty}\langle M(t)>=+\infty$ and by means of the strong law of large numbers (see [17], Section 2.6) we obtain

$$
\lim _{t \rightarrow+\infty} \frac{M(t)}{<M(t)>}=0, \quad \mathbb{P}-\text { a.s. }
$$

Taking into account that, for $t$ large enough,

$$
\frac{|M(t)|}{\ln \lambda(t)}=\frac{|M(t)|}{<M(t)>} \frac{<M(t)>}{\ln \lambda(t)} \leq \frac{|M(t)|}{<M(t)>} \frac{\int_{0}^{t} \varphi_{3}(s) d s}{\ln \lambda(t)} .
$$

We easily deduce now from assumptions (d) that

$$
\lim _{t \rightarrow+\infty} \sup \frac{M(t)}{\ln \lambda(t)}=0, \quad \mathbb{P}-\text { a.s. }
$$

and, consequently,

$$
\lim _{t \rightarrow+\infty} \sup \frac{\ln U(t, X(t))}{\ln \lambda(t)} \leq \theta+\epsilon-\frac{1}{2}(2 \nu-\epsilon)+c, \quad \mathbb{P}-\text { a.s. }
$$

Since the constants $\epsilon>0$ is arbitrary, we can affirm that

$$
\lim _{t \rightarrow+\infty} \sup \frac{\ln \left(|X(t)|-\left(\frac{\varrho}{\lambda(t)^{m}}\right)^{\frac{1}{q}}\right)}{\ln \lambda(t)} \leq-[m-(\theta-\nu+c)], \quad \mathbb{P}-\text { a.s. }
$$

and the proof is therefore complete.

Now, we show an example to illustrate the main results proved in this paper.

Example 3.6. Let us consider the following problem

$$
\left\{\begin{aligned}
d X(t) & =A(t) X(t) d t+g(t, X(t)) d W_{t}, \quad t>0, \\
X(0) & =X_{0}
\end{aligned}\right.
$$

where operators $A(t)$ and $g$ are defined as follows. We consider an open and bounded subset $\mathcal{O} \subset$ $\mathbb{R}^{N}$ with regular boundary and let $2 \leq p<+\infty$. Consider also the Sobolev spaces $V=W_{0}^{1, p}(\mathcal{O})$, $H=L^{2}(\mathcal{O})$ with their usual norms, inner product and duality. The monotone family of operators $A(t): V \rightarrow V^{*}$ is then defined by

$$
<\nu, A(t) u>=-\sum_{i=1}^{N} \int_{\mathcal{O}}\left|\frac{\partial u(x)}{\partial x_{i}}\right|^{p-2} \frac{\partial u(x)}{\partial x_{i}} \frac{\partial \nu(x)}{\partial x_{i}} d x+\int_{\mathcal{O}} \frac{a}{1+t} u(x) \nu(x) d x, \quad \text { for all } u, \nu \in V,
$$

where $a \in \mathbb{R}$, and $g(t, u)=b\left[\frac{t}{1+t}\right]^{\frac{1}{2}} u, b>1, u \in H$ for all $t \in \mathbb{R}_{+}$.

Now, consider the function $U(t, u)=|u|^{2}, u \in H$ and let us compute $L U(t, u)$ and $\mathcal{Q} U(t, u)$. On the one hand, it easily follows

$$
L U(t, u)=2<u, A(t) u>+|g(t, u)|^{2}=-2\|u\|^{p}+\frac{2 a+b^{2} t}{1+t}|u|^{2}, \quad u \in V,
$$


and

$$
L U(t, u) \leq \varphi_{1}(t) U(t, u)+\varrho, \quad \varrho \geq 0
$$

what means that we can set $\varphi_{1}(t)=\frac{2 a+b^{2} t}{1+t}$. On the other hand,

$$
\mathcal{Q} U(t, u)=\left(2 u, b\left[\frac{t}{1+t}\right]^{\frac{1}{2}} u\right)^{2}=4 b^{2} \frac{t}{1+t}|u|^{4},
$$

whence

$$
\varphi_{2}(t) U(t, u)^{2} \leq \mathcal{Q} U(t, u) \leq \varphi_{3}(t) U(t, u)^{2}
$$

and we can choose $\varphi_{2}(t)=\varphi_{3}(t)=4 b^{2} \frac{t}{1+t}, \gamma=0$. Taking $\lambda(t)=e^{t}, m=0, q=2$, we can easily check that the assumptions in the last theorem hold with $\theta=b^{2}, \nu=2 b^{2}, \mu=4 b^{2}$, and therefore

$-[m-(\theta-\nu+1)]=1-b^{2}$. We have then proved practical asymptotic decay to zero with decay function $\lambda(t)=e^{t}, r(t)=\varrho^{\frac{1}{2}}$ and order at least $\gamma=b^{2}-1$.

Remark 3.7. Note that if we take $\lambda(t)=e^{t^{\alpha}}$ and assume that $b^{2}>\alpha$, then, the solution of (3.5) practically asymptotically decays to zero with decay function $\lambda(t)=e^{t^{\alpha}}, r(t)=\varrho^{\frac{1}{2}}$ and order at least $\gamma=b^{2}-\alpha$.

\section{References}

[1] B. Ben Hamed, I. Ellouze, M.A. Hammami, Practical uniform stability of nonlinear differential delay equations. Mediterranean Journal of Mathematics, 8 (2011), no. 4, 603-616.

[2] A. BenAbdallah, M. Dlala, M. A. Hammami, A new Lyapunov function for perturbed non linear systems, Systems and Control letters, 56 (3) (2007) 179-187.

[3] A. BenAbdallah, I. Ellouze and M. A. Hammami, Practical stability of nonlinear time-varying cascade systems, Journal of Dynamical and Control Systems, Vol. 15, No. 1, (2009) 4562.

[4] M. J. Anabtawi, Practical Stability of Nonlinear Stochastic Hybrid Parabolic Systems of Ito-Type: Vector Lyapunov Functions Approach, Journal of Nonlinear Analysis: Real World Applications, Volume 12, Issue 3, (2011) 1386-1400.

[5] M. J. Anabtawi, Almost Sure Convergence Result of Stochastic Parabolic Partial Differential Equations, Journal of Dynamics of Continuous, Discrete and Impulsive Systems, Series A: Mathematical Analysis, 77-88, Volume 15, Number 1, 2008. (Waterloo University Press).

[6] M. J. Anabtawi, S. Sathananthan, Stability and Convergence Results for Ito-Type Parabolic Partial Differential Equations in Hilbert Spaces, Journal of Stochastic Analysis and Applications, Volume 27, Issue 4, 2009, Pages 671-693.

[7] L. Arnold, Stochastic differential equations: Theory and Applications, John Wiley, New York-London-Sydney, 1974.

[8] L. Arnold, A formula connecting sample and moment stability of linear stochastic systems, SIAM J.Appl.Math, 44 (1984), 793-802. 
[9] L. Arnold, E.Oeljelaus and E. Pardoux, Almost sure and moment stability for linear Itô equations, Lyapunov Exponents (Bremen, 1984), 129-159, Lecture Notes in Math. 1186, SpringerVerlag, Berlin-New York, 1986.

[10] T. Caraballo, 1991. Existence and uniqueness of solutions for nonlinear stochastic partial differential equations, Collectanea Mathematica, 42 (1): 5174.

[11] T. Caraballo, On the decay rate of solutions of non-autonomous differential systems, Electron. J. Diff. Eqns. 2001, 2001 (05), 1-17.

[12] T. Caraballo, Asymptotic exponential stability of stochastic partial differential equations with delay, Stochastics 1990, 33, 27-47.

[13] T. Caraballo, K. Liu, On exponential stability criteria of stochastic partial differential equations, Stochastic Proc. Appl. 1999, 83, 289-301.

[14] T. Caraballo, Maria J. Garrido-Atienza, José Real, Asymptotic Stability of Nonlinear Stochastic Evolution Equations, Stochactic Analysis and Applications, Vol .21, No. 2, 301$327,2003$.

[15] T. Caraballo, Maria J. Garrido-Atienza, José Real, Stochastic stabilization of differential systems with general decate rate, Systems and Control Letters, 48 (2003), 397-406.

[16] T. Caraballo, M. A. Hammami, L. Mchiri, On the global uniform asymptotic stability of stochastic differential equations, Preprint.

[17] R. Z. Has'minskii, Stochastic Stability of Differential Equations, Sijthoff Noordhoff, 1980.

[18] U. G. Haussmann, Asymptotic stability of the linear Itô equation in infinite dimensions, $J$. Math. Anal. Appl. 1978, 65, 219-235.

[19] H. K. Khalil, Nonlinear Systems, Mac-Millan, 2nd edition, 1996.

[20] R. Sh. Lipster, A. N. Shirayev, Theory of Martingales, Dordrecht. Kluwer Academic Publ Dordrecht, The Netherlands, (1989).

[21] K. Liu, X. R. Mao, Large time decay behavior of dynamical equations with random perturbation features, Stochastic Anal. Appl. 19 (2) (2001) 295-327.

[22] K. Liu, A. Truman, A stability of Stochastic Differential Equations In Infinite Dimensions, Monograph.

[23] X. Mao, Almost sure polynomial stability for a class of SDEs, Quart. J. Math. Oxford Ser. (2) 43 (1992) 339-348.

[24] X. Mao, Exponential Stability of Stochastic Differential Equations, Marcel Dekker, Inc.: New York, 1994.

[25] X. Mao, Stochastic Differential Equations and Applications, Ellis Horwood, Chichester, U.K, 1997. 
[26] E. Pardoux, Équations aux Dérivées Partielles Stochastiques Non Linéaires Monotones, Thèse, Université Paris XI, 1975, 236.

[27] U. H. Thygesen, A survey of Lyapunov Techniques for Stochastic Differential Equations, IMM Technical Report n.c, 1997. 